\title{
FOR HOW MANY EDGES IS A DIGRAPH ALMOST CERTAINLY HAMILTONIAN?
}

\author{
E. M. WRIGHT ${ }^{1}$
}

\begin{abstract}
K_{n}(r)$ is the number of those Hamiltonian cycles in the complete digraph on $n$ nodes, each of which has just $r$ edges in common with a particular Hamiltonian cycle, and $B(n, r)=$ $n ! /\{r !(n-r) !\}$. We show that $K_{n}(r)=B(n, r) K_{n-r}(0)$ and deduce that $K_{n}(0) \sim(n-1) ! e^{-1}$ for large $n$ and that $K_{n}(r) \sim(n-1) ! e^{-1} / r !$ if $r=o(n)$. An $(n, q)$ digraph is one with $n$ labelled nodes and $q$ edges. From the result for $K_{n}(r)$, we deduce that, if $q n^{-3 / 2} \rightarrow \infty$ as $n \rightarrow \infty$, then almost all $(n, q)$ digraphs are Hamiltonian. If $q n^{-3 / 2} \rightarrow c$ as $n \rightarrow \infty$, then the proportion of $(n, q)$ digraphs which are nonHamiltonian is at most $1-\exp \left(-c^{-2}\right)$ as $n \rightarrow \infty$.
\end{abstract}

An $(n, q)$ digraph consists of $n$ nodes and $q$ directed edges. We consider only labelled digraphs, i.e. digraphs whose nodes are labelled. If $A, B$ are two different nodes, they may be not joined or joined by the edge $A B$, or by the different edge $B A$ or by both $A B$ and $B A$. The greatest possible number of edges is $N=n^{2}-n$ and the $(n, N)$ digraph is called the complete digraph. We write $B(h, k)=h ! /\{k !(h-k) !\}$, the usual binomial coefficient. Then obviously there are just $B(N, q)$ different $(n, q)$ digraphs. We call a sequence of $n$ edges

$$
A_{1} A_{2}, A_{2} A_{3}, A_{3} A_{4}, \cdots, A_{n-1} A_{n}, A_{n} A_{1},
$$

(where $A_{1}, \cdots, A_{n}$ are all the different $n$ nodes of the graph) a Hamiltonian cycle (H.c.) and we say that a digraph which contains such a cycle is Hamiltonian. Our principal object is to find (so far as we can) for what $q$ almost all $(n, q)$ graphs are Hamiltonian.

We write $K_{n}(r)$ for the number of H.c. in the complete graph, each of which has just $r$ edges in common with the specified H.c. in (1). The asymptotic approximation to $K_{n}(r)$ for large $n$ and appropriate $r$ is essential to our method, which is otherwise that used by O'Neil [2] to study the

Presented to the Society January 8, 1973 under the title Hamiltonian graphs and digraphs; received by the editors January 10, 1973.

AMS (MOS) subject classifications (1970). Primary 05C20.

Key words and phrases. Digraph, Hamiltonian cycle.

1 The research reported herein was sponsored in part by the United States Government.

(c) American Mathematical Society 1973 
permanents of $(0,1)$ matrices. In his case, the number $K_{n}(r)$ is replaced by $D_{n}$, Euler's rencontre number, whose asymptotic approximation, viz. $D_{n}=n ! e^{-1}+O(1 / n)$, was found by Euler [1]. I find here an exact value for $K_{n}(r)$, which is perhaps of some interest, but only the asymptotic approximation is needed for my main results.

If we apply the method used here to study Hamiltonian graphs, the number $K_{n}(r)$ is replaced by $H_{n}(r)$. I can find an asymptotic approximation to $H_{n}(r)$ for large $n$ when $r^{2}=o(n)$ and this is enough to prove my principal result about Hamiltonian graphs. But the study of $H_{n}(r)$ is more troublesome than that of $K_{n}(r)$, so I postpone this. Palasti [3] has calculated $H_{n}(n-2), H_{n}(n-3)$ and $H_{n}(n-4)$ and found other results about $H_{n}(r)$. These illustrate the relative complexity of $H_{n}(r)$ compared with $K_{n}(r)$.

Obviously $K_{n}(n)=1, K_{n}(n-1)=0$ and $K_{3}(0)=1$. We define $K_{0}(0)=1$, $K_{1}(0)=0$. We prove first that

$$
K_{n}(r)=B(n, r) K_{n-r}(0)
$$

Clearly (2) is true for $r=n$ and $r=n-1$. We suppose $r \leqq n-2$. We can choose the set of $r$ edges out of the $n$ edges in (1) in just $B(n, r)$ ways. Take any one such set. In the cycle (1) contract each of these $r$ edges (or each consecutive run of them) and their end-nodes into a special node. We are left with an H.c. on $n-r$ nodes, of which $r$ or less are special. In the complete digraph on $n-r$ nodes there are just $K_{n-r}(0)$ different H.c. which have no edges in common with our H.c. contracted from (1). If in each of these we expand each special node appropriately (i.e. by replacing it by the nodes and edge or edges from which it was contracted), we have an H.c. in the complete graph on $n$ nodes which has just $r$ edges in common with the H.c. in (1). There is no ambiguity about how a special node is replaced by the contracted edge or edges, since the direction of the new H.c. determines this in just one way. Thus the number of H.c. in the complete graph on $n$ nodes which has just the prescribed set of $r$ edges in common with the H.c. in (1) is $K_{n-r}(0)$. The result (2) follows.

There are just $(n-1)$ ! different H.c. in the complete digraph on $n$ nodes and so

$$
(n-1) !=\sum_{r=0}^{n} K_{n}(r) .
$$

If we use (2) and put $k_{0}=1, k_{1}=0$ and $k_{n}=K_{n}(0) / n$ ! when $n \geqq 2$, (3) becomes

$$
1 / n=\sum_{r=0}^{n} k_{n-r} / r !
$$


where, as usual, $0 !=1$. Hence, if $|X|<1$ and $W(X)=\sum_{n=0}^{\infty} k_{n} X^{n}$, we have

and so

$$
1+\sum_{n=1}^{\infty} X^{n} / n=e^{X_{W}} W(X)
$$

Hence, for $n \geqq 1$,

$$
W(X)=e^{-X}\left(1+\sum_{n=1}^{\infty}\left(X^{n} / n\right)\right)
$$

and so

$$
k_{n}=\sum_{r=0}^{n-1}(-1)^{r} /\{r !(n-r)\}+(-1)^{n} / n !
$$

Hence

$$
K_{n}(0)=n ! \sum_{r=0}^{n-1}(-1)^{r} /\{r !(n-r)\}+(-1)^{n}
$$

$$
K_{n}(0)+K_{n-1}(0)=(n-1) ! \sum_{r=0}^{n-1}(-1)^{r} / r !=D_{n-1},
$$

where $D_{n-1}$ is Euler's rencontre number [1]. By (3), $K_{n-1}(0) \leqq(n-2)$ !, and so, since $D_{n-1} \sim(n-1) ! e^{-1}$, we have

$$
K_{n}(0) \sim(n-1) ! e^{-1} .
$$

Before using (5) for our main purpose, we remark that (4) gives us

since $K_{3}(0)=1$.

$$
K_{n}(0)=\sum_{s=1}^{n-3}(-1)^{s-1} D_{n-s}+(-1)^{n-1},
$$

We now denote by $\pi$ any H.c. in the complete digraph on $n$ nodes; $\pi$ is associated with just one permutation of the nodes $A_{2}, A_{3}, \cdots, A_{n}$ in (1). For any particular digraph we write $E_{\pi}=1$, if the digraph contains $\pi$, and $E_{\pi}=0$, if not. We write $E=\sum_{\pi} E_{\pi}$, so that the digraph is or is not Hamiltonian according as $E>0$ or $E=0$. It is clear that $\pi$ occurs in just $B(N-n, q-n)$ of the $(n, q)$ digraphs. Hence, if $\Sigma^{\prime}$ denotes summation over all $(n, q)$ digraphs, we have

$$
\sum^{\prime} E=\sum_{\pi} \sum^{\prime} E_{\pi}=(n-1) ! B(N-n, q-n)
$$

and so $M(E)$, the average value of $E$ over all $(n, q)$ digraphs, is

$$
M(E)=(n-1) ! B(N-n, q-n) / B(N, q) .
$$

Again $\sum^{\prime} E^{2}=\sum_{\pi} \sum^{\prime} E_{\pi}^{2}+\sum_{\pi \neq \pi^{\prime}} \sum^{\prime} E_{\pi} E_{\pi^{\prime}}$. If $\pi, \pi^{\prime}$ have just $r$ edges in common, they occur together in just $B(N-2 n+r, q-2 n+r)$ of the $(n, q)$ digraphs and so $\sum^{\prime} E_{\pi} E_{\pi^{\prime}}=B(N-2 n+r, q-2 n+r)$. But there are 
just $(n-1) ! K_{n}(r)$ pairs $\pi, \pi^{\prime}$ (order relevant) which have just $r$ edges in common and so

$$
\sum^{\prime} E^{2}=(n-1) ! \sum_{r=0}^{n} K_{n}(r) B(N-2 n+r, q-2 n+r) .
$$

For a non-Hamiltonian digraph, we have $E=0$ and so $(E-M(E))^{2}=$ $(M(E))^{2}$. Hence the number of non-Hamiltonian $(n, q)$ digraphs is not greater than $\sum^{\prime}\{E-M(E)\}^{2} /\{M(E)\}^{2}$. But $M\{E-M(E)\}^{2}=M\left(E^{2}\right)-$ $\{M(E)\}^{2}$ and so the proportion of $(n, q)$ digraphs which are not Hamiltonian is not greater than $\left[M\left(E^{2}\right) /\{M(E)\}^{2}\right]-1$. Now

where

$$
\frac{M\left(E^{2}\right)}{\{M(E)\}^{2}}=\frac{B(N, q) \sum^{\prime} E^{2}}{\left(\Sigma^{\prime} E\right)^{2}}=\sum_{r=0}^{n} \frac{\omega(r) K_{n}(r)}{(n-1) !},
$$

$$
\begin{aligned}
\omega(r) & =B(N, q) B(N-2 n+r, q-2 n+r)\{B(N-n, q-n)\}^{-2} \\
& =\prod_{s=0}^{n-1}\left(\frac{N-s}{q-s}\right) \prod_{s=0}^{n-r-1}\left(\frac{q-n-s}{N-n-s}\right) .
\end{aligned}
$$

We now take $q=n^{3 / 2} \psi$, where $\psi=\psi(n) \rightarrow \infty$ as $n \rightarrow \infty$. Since the proportion of $(n, q)$ digraphs which are Hamiltonian is nondecreasing as $q$ increases we may without loss of generality suppose that $\psi<\log n$. We have

$$
\begin{aligned}
\log \omega(r) & =r \log (N / q)+\sum^{\prime \prime}(\log \{1-(s / N)\}-\log \{1-(s / q)\}) \\
& =r \log (N / q)+\left(q^{-1}-N^{-1}\right) \sum^{\prime \prime} s+o(1),
\end{aligned}
$$

where

$$
\Sigma^{\prime \prime} s=\left(\sum_{s=0}^{n-1}-\sum_{s=n}^{2 n-r-1}\right) s=-n^{2}+2 n r-\frac{1}{2} r^{2}+o(q) .
$$

Hence, for $r \leqq n^{5 / 8}$ (say), we have

$$
\log \omega(r)=r \rho-\left(n^{2} / q\right)+1+o(1),
$$

where $\rho=\rho(n, q)=\log (N / q)+(2 n / q)$, while, for $r>n^{5 / 8}$, we have

$$
\log \omega(r) \leqq r \rho-\left(n^{2} / q\right)+1+o(1) .
$$

Hence, if we put $m=3\left[e^{\rho}\right]$, we see that $\sum_{r=m+1}^{n} e^{r \rho} / r !=o(1)$ and

$$
\begin{aligned}
M\left(E^{2}\right)\{M(E)\}^{-2} & \sim \exp \left(-n^{2} q^{-1}\right)\left\{\sum_{r=0}^{m}\left(e^{r \rho} / r !\right)+o(1)\right\} \\
& \sim \exp \left\{e^{\rho}-\left(n^{2} q^{-1}\right)\right\},
\end{aligned}
$$


where $e^{\rho}-n^{2} q^{-1}=\left(N e^{2 n / q}-n^{2}\right) / q=o(1)$. Hence $M\left(e^{2}\right)\{M(e)\}^{-2} \sim 1$ and so the proportion of non-Hamiltonian $(n, q)$ digraphs is $o(1)$.

We have thus proved the following theorem.

THEOREM 1. If $q^{-3 / 2} \rightarrow \infty$ as $n \rightarrow \infty$, then almost all labelled $(n, q)$ digraphs are Hamiltonian.

If we take our expansion of $\log \omega(r)$ to one further term, i.e. consider $\Sigma^{\prime \prime} s^{2}$, we can prove the following theorem.

THEOREM 2. If $q^{n^{-4 / 3} \rightarrow \infty}$ as $n \rightarrow \infty$ then $M\left(e^{2}\right)\{M(e)\}^{-2} \sim \exp \left(n^{3} q^{-2}\right)$.

This shows that we cannot improve Theorem 1 by our method. But it is very unlikely that the result is best possible. A little calculation however enables us to prove the following result.

THEOREM 3. If $q^{-3 / 2} \rightarrow c$ as $n \rightarrow \infty$, then the proportion of non-Hamiltonian labelled $(n, q)$ digraphs is not more than $1-e^{-c^{-2}}$ in the limit.

\section{REFERENCES}

1. L. Euler, Calcul de la probabilité dans le jeu de rencontre, Mém. Acad. Sci. Berlin 7 (1753), 255-270; Opera omnia (1) 7 (1923), 11-25.

2. P. E. O'Neil, Asymptotics in random $(0,1)$ matrices, Proc. Amer. Math. Soc. 25 (1970), 290-296. MR 41 \#92.

3. I. Palasti, On the common edges of Hamilton-cycles of a complete linear graph, Combinatorial Theory and its Applications III (Colloq. Math. Soc. János Bolyai, 4), Amsterdam, 1970, pp. 829-868.

Office of the Principal, University of Aberdeen, Aberdeen, United Kingdom 